\title{
Den Alltag gesünder gestalten
}

\section{Gesundheit fördern bedeutet: Befähigung zur Gestaltung gesunder Lebenswelten}

\author{
Rike Hertwig und Holger Kilian
}

Rike Hertwig, M.A. ist Referentin für Öffentlichkeitsarbeit bei Gesundheit Berlin-Brandenburg e. V.

E-Mail hertwig@gesundheitberlin.de Dipl.-Soz. Holger Kilian, MPH ist Referent für Gesundheitsförderung bei Gesundheit Berlin-Brandenburg e. V. mit den Arbeitsschwerpunkten Gesundheit und soziale Lage, Gesundheitsberichterstattung, Gesundheitsziele und Qualitätsentwicklung.

E-Mail kilian@gesundheitberlin.de
Menschen in schwieriger sozialer Lage sind durch Informationsmaterialien oder Kursangebote schwer erreichbar. Ein nachhaltiger Erfolg hängt maßgeblich von der Beeinflussung des Verhaltenskontextes und der konkreten Lebensbedingungen $a b$.

Gesundheitschancen sind sozial ungleich verteilt: Menschen mit niedrigem Bildungsstand, geringem Einkommen, besonderen sozialen Belastungen oder Ausgrenzungen sind überdurchschnittlich häufig von Krankheit betroffen. Sie haben zudem eine geringere Lebenserwartung als sozial Bessergestellte. Die Gesundheitsberichterstattung des Bundes, aber auch der Länder und Kommunen liefern hierzu vielfache Belege und Hintergründe. Sowohl materielle, als auch psychosoziale Faktoren spielen ursächlich eine Rolle: Belastende Lebensbedingungen, oft auch ein riskanteres Gesundheitsverhalten, summieren sich im Lebensverlauf und führen zu schlechteren Gesundheitschancen.

Diese Gesundheitsgefährdungen und Gesundheitsbelastungen lassen sich durch rein verhaltensorientierte Maßnahmen schwer verringern. Sie resultieren aus komplexen und sich zum Teil verstärkenden Belastungsfaktoren. Hinzu kommt häufig ein belastendes Gesundheitsverhalten, das Teil des individuellen Lebensstils ist, bzw. Teil der Bewältigungsstrategie, um den alltäglichen Belastungen zu begegnen. Geringes Einkommen, eine schwierige Wohnsituation, Sorgen in der Familie, Unsicherheit, Ausgrenzung, Arbeitslosigkeit, aber auch ein hohes Verkehrsaufkommen im Stadtteil und weitere Umweltfaktoren sind Teil der komplexen Determinanten für Gesundheit (Wilkinson und Marmot, 2004). In den Städten kumulieren diese benachteiligten Lebenslagen häufig in einigen Stadtteilen, für Berlin belegt dies zum Beispiel der Berliner Sozialstrukturatlas (SenGuV 2009).
Wie die Forschung zeigt, sind Menschen in schwieriger sozialer Lage über Informationsmaterialien oder Kursangebote schwer erreichbar, ein nachhaltiger Erfolg hängt maßgeblich von der Beeinflussung des Verhaltenskontextes und der konkreten Lebensbedingungen ab (nach Rosenbrock 2008). So verdeutlicht das Regenbogenmodell von Whitehead und Dahlgren, dass der individuelle Lebensstil nur ein Einflussfaktor auf die Gesundheit ist, denn den konkreten Lebens- und Arbeitsbedingungen, dem sozialen Umfeld, aber auch der Rechtsprechung sowie den wirtschaftlichen, kulturellen und politischen Rahmenbedingungen kommt ebenfalls eine große Bedeutung zu (vgl. Abb. Seite 210).

Gesundheitsschutz ist vor diesem Hintergrund mehr als nur der defensive Schutz vor den klassischen Gesundheitsgefährdungen wie zum Beispiel Unfällen oder Schadstoffen in Lebensmitteln. Ebenso muss er die gesamten Lebensumstände in den Blick nehmen, um in einer umfassenden Perspektive die dort liegenden gesundheitlichen $\mathrm{Ri}$ siken zu vermindern und Potenziale zu entwickeln. Hier gibt es viele Ressourcen, die zur Verbesserung der Gesundheitschancen aktiviert werden können, beispielsweise Informationen, Bildung, Handlungswissen, Partizipation, Verhaltensspielräume oder soziale Unterstützung.

Diese Ressourcen gezielt zu stärken und die Menschen zu befähigen, die gesundheitsrelevanten Rahmenbedingungen ihres Lebensumfeldes selbst aktiv zu gestalten, dies ist das Ziel der Gesundheitsförderung. Im Mainzer Projekt »Gesundheit jetzt - in sozialen Brennpunkten « beispielsweise arbeiten die Bewohnerinnen und Bewohner einer Obdachlosensiedlung gemeinsam daran, bessere Lebensbedingungen für Kinder, Jugendliche und ihre Familien zu schaffen. (Weitere Informationen zu dem Projekt können im Internet eingesehen werden: http://www.gesundheitliche-chancengleichheit.de/:datenbank.) 


\section{Gesundheitsbelastungen senken - Gesundheitspotenziale stärken}

Gesundheitsförderung verfolgt den doppelten Ansatz, zum einen Gesundheitsbelastungen zu senken und gleichzeitig die individuellen Gesundheitspotenziale zu stärken. Der Stadtteil ist dabei ein wichtiges Setting, um insbesondere sozial benachteiligte Menschen zu erreichen. So werden in den Quartieren des Bund-Länder-Programms »Soziale Stadt « (www. sozialestadt.de) viele Angebote und Maßnahmen entwickelt, welche u. a. die sozialen Netzwerke in der Lebenswelt stärken und gleichzeitig einen positiven Beitrag zur Gesundheit der Bewohnerinnen und Bewohner leisten. Konkret bedeutet dies, dass im Rahmen interprofessioneller Kooperationen beispielsweise der Bereiche Gesundheit, Stadtentwicklung, Verkehr und Bildung die gesundheitsfördernden Bedingungen im Stadtteil verbessert werden. Durch aktivierende Ansätze werden die Kompetenzen von Einzelnen und Gemeinschaften gestärkt, sich im Sinne eines Empowerment aktiv für die eigenen gesundheitlichen Belange einzusetzen. Die Interventionen in Lebenswelten tragen auf diese Weise dazu bei, dass die Gesundheit aller dort lebenden Personen gefördert wird.

Bei den Interventionen im Stadtteil kommt der Zusammenarbeit von Gesundheitsförderung und Sozialer Arbeit eine zentrale Bedeutung zu. Im Rahmen beispielsweise von Quartiersmanagements können Themen wie altersangemessene Bewegungsangebote, gesunde Ernährung und soziale Unterstützung thematisiert und im Rahmen gemeinwesenorientierter Angebote umgesetzt werden. Sozialarbeiterinnen und Sozialarbeiter verfügen durch ihre Arbeit vor Ort über vielfältige Zugänge zu Menschen in schwieriger sozialer Lage. Dies sind vor allem Alleinerziehende, sozial benachteiligte Familien mit Kindern rund um die Geburt sowie Arbeitslose und Seniorinnen und Senioren. Eine verstärkte Integration von Gesundheitsförderung und Prävention in die soziale Arbeit mit Kindern und Jugendlichen ist das Schwerpunktthema des 13. Kinder- und Jugendberichtes (BMFSFJ 2009).

Wie Gesundheitsförderung im Stadtteil gelingt, stellen die Arbeitsmaterialien »Aktiv werden für Gesundheit - Arbeitshilfen für Prävention und Gesundheits- förderung im Quartier « dar. Sie geben zudem Tipps für Projektanträge und Projektfinanzierung und liefern Checklisten. Die Arbeitshilfen wurden im Auftrag des Bundesministeriums für Gesundheit (BMG) im Rahmen von IN FORM, Deutschlands Initiative für gesunde Ernährung und mehr Bewegung, erstellt. Sie wurden durch den Kooperationsverbund Gesundheitsförderung bei sozial Benachteiligten entwickelt und helfen das Thema Gesundheitsförderung im Bund-LänderProgramm Soziale Stadt langfristig zu verankern.
2005 ins Leben gerufen, um in Modellkommunen durch die Integration von Gesundheitsförderung, Prävention und Rehabilitation eine Neuausrichtung der Seniorenpolitik zu initiieren. Die Stadt will vor allem die Älteren einbeziehen, richtet sich darüber hinaus aber auch mit einem Gesamtkonzept an alle Bürgerinnen und Bürger der Stadt, insbesondere sozial Benachteiligte. NAIS ist im Programmgebiet Soziale Stadt aktiv, wo beispielsweise Quartiersmanagements in die Koordination von präventiven Hausbesuchen und deren Verknüpfung mit Be-

\section{»Mehr Handlungsspielräume, ein kooperatives Arbeitsklima und Austauschmöglichkeiten haben eine zentrale Bedeutung für eine gesunde Arbeitssituation "}

\section{Aktivierung von Ressourcen vor Ort}

Im Rahmen des von der Bundeszentrale für gesundheitliche Aufklärung (BZgA) initiierten und unterstützten bundesweiten Kooperationsverbundes »Gesundheitsförderung bei sozial Benachteiligten « (www.gesundheitliche-chancengleichheit.de) werden in einer umfangreichen Praxisdatenbank mehr als 100 Beispiele guter Praxis gesammelt und vorgestellt, die solche Ansätze erfolgreich umsetzen. Dabei sind zahlreiche Angebote vertreten, die eine gelungene interprofessionelle $\mathrm{Zu}$ sammenarbeit im Hinblick auf unterschiedliche Zielgruppen aufzeigen. Die Zusammenarbeit mit Professionellen aus der Sozialen Arbeit und den sozialpflegerischen Diensten spielt dabei eine große Rolle.

So gibt es Good-Practice-Projekte, die im Stadtteil angesiedelt sind und insbesondere auf ein integriertes Handlungskonzept und Vernetzung sowie Partizipation setzen.

Dazu gehört beispielsweise das Projekt "Neues Altern in der Stadt « (NAIS) aus Bruchsal. Dieses Projekt wurde im Jahr wegungs- und anderen Angeboten eingebunden sind. Die Beteiligung der Bürgerinnen und Bürger wird $u$. a. darüber sichergestellt, dass diese im Rahmen einer Zukunftswerkstatt Probleme und Bedürfnisse diskutierten und Lösungsideen entwickelten. Aus der Analysephase wurden schließlich Projektideen entwickelt. Dazu gehört zum Beispiel ein Mehrgenerationenhaus in der Bruchsaler Kernstadt, die Projekte »Fit bis ins hohe Alter «, »Pflegebedürftigkeit geht mich an «, ein Bürgerhaus sowie ein Seniorenwegweiser. Weiterhin werden generationenübergreifende Koch- und Bewegungsprogramme realisiert. Das Projekt hat zahlreiche Kooperationspartner an einen Tisch gebracht, die gemeinsam Projekte entwickeln und durchführen. Beteiligt sind beispielsweise Wohlfahrtsverbände, städtische Firmen, Ärztinnen und Ärzte, Krankenkassen, Schulen, Sportvereine und Seniorengremien.

Ein weiteres Projekt, dass ebenfalls im Setting Stadtteil durchgeführt wird und für Partizipation sowie integriertes Handlungskonzept und Vernetzung als gute Praxis beschrieben wurde, ist das »Marburger Gesundheitsnetzwerk Mit- 


\section{Arbeitshilfen für die Praxis}

Die Dokumentation »Gesundheitsförderung im Stadtteil « liefert konkretes Anschauungsmaterial, wie gesundheitsfördernde Strategien im Quartier kooperativ und partizipativ entwickelt und umgesetzt werden können. Die Sammlung bietet konkrete Beispiele, geht aber auch auf übergreifende Zusammenhänge von Gesundheit und Sozialer Lage ein.

Internet http://www.gesundheitliche-chancengleichheit.de/:arbeitshilfen

tendrin ", das vor allem in drei sozial benachteiligten Stadtteilen von Marburg aktiv ist. Klettern, Hangeln, Balancieren, Springen und Laufen - diese Bewegungsmöglichkeiten sollen für Kinder in ihrem Wohnumfeld erfahrbar sein. Sie werden ermuntert, ihren Sozialraum für freies Bewegungsspiel zu nutzen. Auch gemeinsames Kochen und interkulturelle Essgewohnheiten werden thematisiert. Dazu haben sich über 40 Netzwerkpartner aus den Bereichen der Sozialen Arbeit, Verwaltung, Gesundheit, Medien und Wissenschaft $\mathrm{zu}$ einem interdisziplinären Netzwerk zusammengeschlossen. Zielgruppe sind Kinder bis zehn Jahren und deren Familien. Der sozialräumliche Zugang zu den Lebenswelten der Kinder und ihrer Eltern steht im Vordergrund. Träger ist der Mar- burger Verein für bewegungs- und sportorientierte Jugendsozialarbeit e. V. (bsj). Das Netzwerk geht davon aus, dass kommunale Gesundheitsförderung nur gemeinsam mit allen Akteuren des Gemeinwesens wirkungsvoll umgesetzt werden kann.

\section{Gesundheitsressourcen der Beschäftigten in der Sozialen Arbeit stärken}

Der Settingansatz in Kombination mit einem integrierten Handlungskonzept, Vernetzung und Partizipation stellt eine gute Grundlage dar, um Aktivitäten im Stadtteil erfolgreich zu gestalten und die Gesundheitsressourcen benachteiligter Menschen im Stadtteil zu stärken. Doch wie sieht es mit den Professionellen in der Sozialen Arbeit und den sozialpflegerischen Diensten aus?

Eine Untersuchung zur gesundheitlichen Situation von Frauen in Deutschland im Auftrag des Bundesministeriums für Jugend, Familie, Senioren und Frauen (BMJFSF, 1999), die unter anderem die gesundheitliche Situation von Frauen in sozialpflegerischen Berufen untersucht hat, verweist auf hohe psychische Belastungen dieser Berufsgruppen durch Verantwortungsdruck oder auch widersprüchliche Anforderungen. Die hohe Personenbezogenheit erfordere in diesen Berufen, sich mit der ganzen Persönlichkeit in die Arbeit einzubringen. Ebenfalls seien ein hoher Zeitdruck und geringe Handlungsspielräume Bestandteil der Arbeit. Diese Merkmale der Tätigkeit in sozialpflegerischen Berufen sind Bedingungen, die Stress auslösen können.

Ansätze der Gesundheitsförderung in Lebenswelten, die mit einem integrierten Handlungskonzept arbeiten und Vernetzung initiieren, können auch die Ressourcen der Professionellen stärken. Kooperationen über die Professionsgrenzen hinweg ermöglichen den Professio-

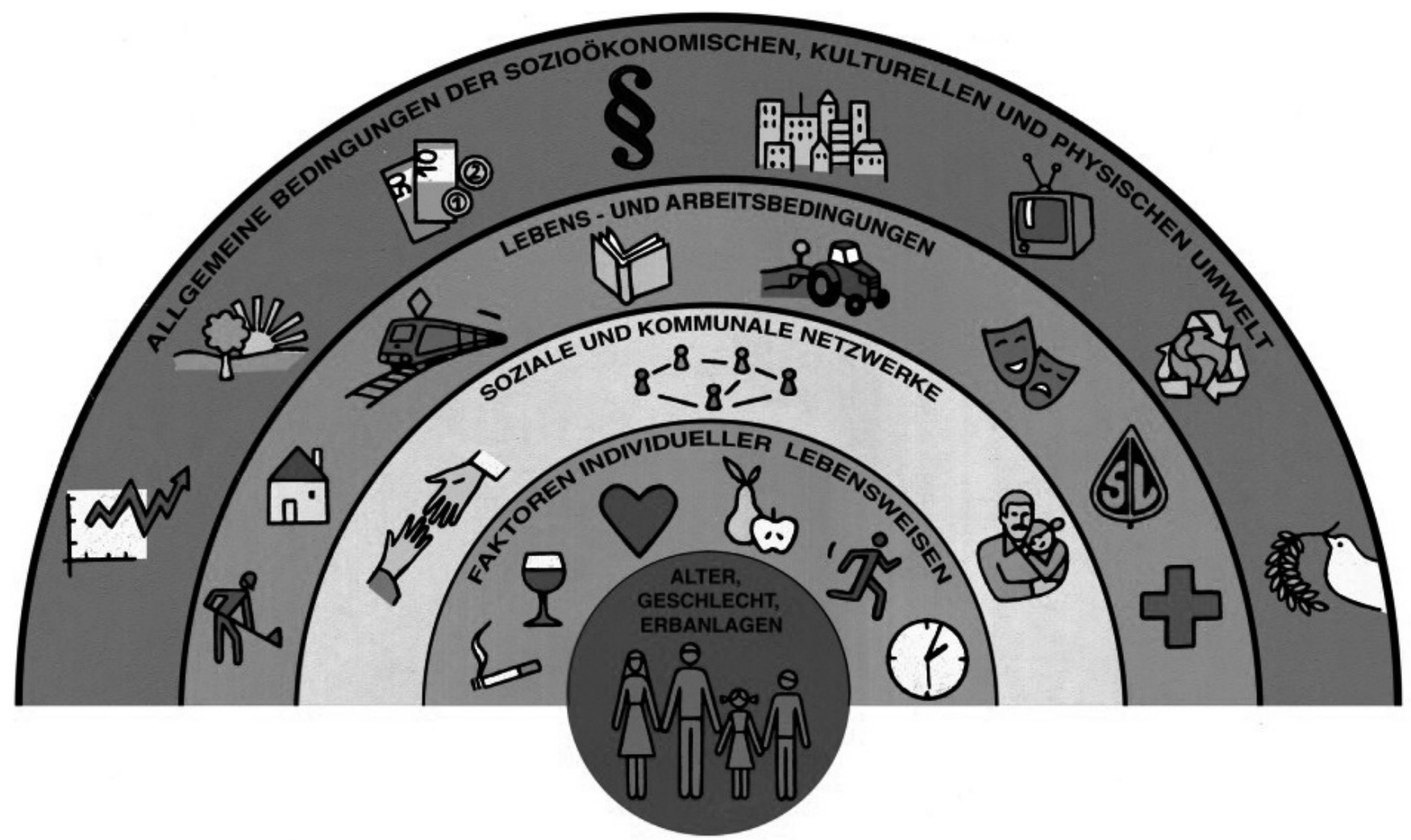

Das Regenbogenmodell fasst die Determinanten für Gesundheit grafisch zusammen. Danach ist der individuelle Lebensstil nur einer der Einflussfaktoren für die Gesundheit. (Abbildung nach Whitehead und Dahlgren 1991, Darstellung: Fonds Gesundes Österreich) 
nellen aus der Sozialen Arbeit ein erweitertes Handlungsspektrum, Kooperationen und Austausch. So hat der Bericht des Bundesfamilienministeriums herausgearbeitet, dass unter anderem die Erweiterung von Handlungsspielräumen sowie kooperatives und unterstützendes $\mathrm{Ar}$ beitsklima und Austauschmöglichkeiten eine zentrale Bedeutung für eine verbesserte Arbeitssituation in sozialpflegerischen Berufen haben.

\section{Literatur}

BMFSFJ (Bundesministeriums für Familie, Senioren, Frauen und Jugend) (Hg.) 2009: Bericht über die Lebenssituation junger Menschen und die Leistungen der Kinder- und Jugendhilfe in Deutschland, 13. Kinder- und Jugendbericht, Bundestags-Drucksache 16/12860 vom 30.04.2009. Berlin.

BMJFSF (Bundesministerium für Jugend, Familie, Senioren und Frauen) 1999: Bericht zur gesundheitlichen Situation von Frauen in Deutschland. Eine Bestandsaufnahme unter Berükksichtigung der unterschiedlichen Entwicklung in West- und Ostdeutschland. Berlin. Kohlhammer.

Rosenbrock, R. 2008: Primärprävention - Was ist das und was soll das?, Discussion Papers der Forschungsgruppe Public Health März 2008. Berlin: Wissenschaftszentrum Berlin für Sozialforschung. Internet http://bibliothek.wzberlin.de/pdf/2008/i08-303.pdf.

SenGuV (Senatsverwaltung für Gesundheit, Umwelt und Verbraucherschutz Berlin) (Hg.) 2009: Sozialstrukturatlas 2008, Ein Instrument der quantitativen, interregionalen und intertemporalen Sozialraumanalyse und -planung. Berlin: Senatsverwaltung für Gesundheit, Umwelt und Verbraucherschutz. Internet http://www.berlin.de/sen/statistik/gessoz/gesundheit/spe zial.html.

Whitehead, M.; Dahlgren, G. 1991: What can we do about inequalities in health? in: Lancet 338, 1059-1063.

Wilkinson, R.; Marmot, M. (Hrsg.) 2004: Soziale Determinanten von Gesundheit - Die Fakten, zweite Ausgabe. Kopenhagen: WHO. Internet http://www.euro.who.int/document/e81 384 g.pdf.
Vor diesem Hintergrund lässt sich argumentieren, dass diese Art präventiver Maßnahmen tatsächlich für alle Beteiligten zu einer gesundheitsförderlichen Lebenswelt beiträgt.

\section{Fazit}

In gelungenen gesundheitsfördernden Projekten und Maßnahmen ist »Gesund- heitsschutz « nicht eine von oben initiierte und von den »Betroffenen « passiv empfangene Wohltat, sondern das Ergebnis eines gemeinsam von Professionellen und betroffenen Menschen getragenen Prozesses. Die erfolgreiche Umsetzung solcher Prozesse erfordert Engagement und langen Atem, ist aber aus Sicht der Gesundheitsförderung der erfolgversprechendste Weg, nachhaltig Gesundheit und Gesundheitsschutz im Alltag zu verankern.

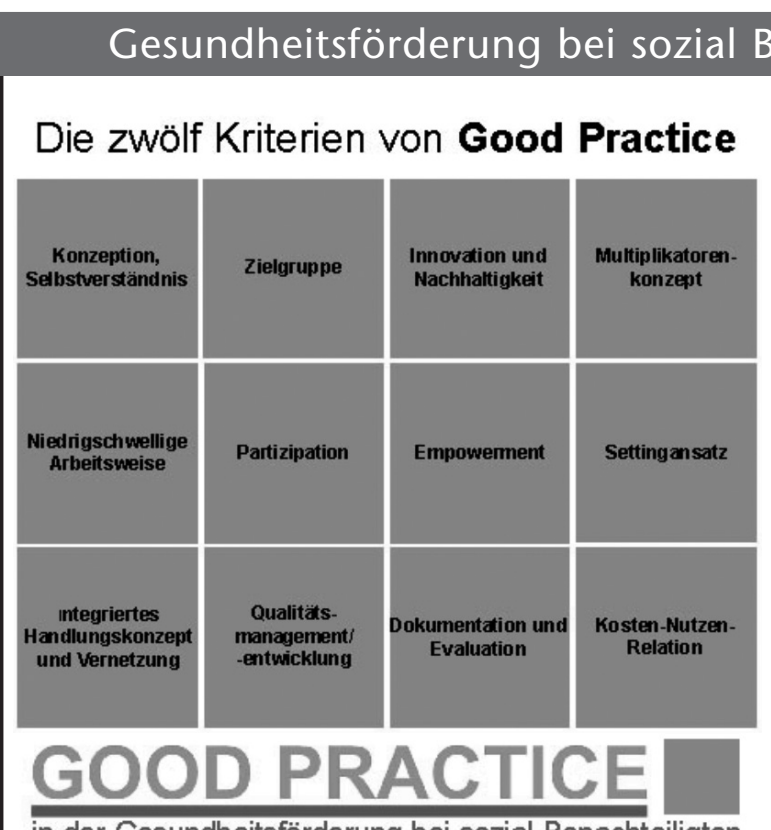

in der Gesundheitsförderung bei sozial Benachteiligten des » Gesundheitsförderung bei sozial Benachteiligten « fortgeführt, dem inzwischen 53 Kooperationspartner angehören.

Herzstück der Plattform ist eine online recherchierbare bundesweite Datenbank für gesundheitsfördernde Projekte und Angebote, die im Auftrag der Bundeszentrale für gesundheitliche Aufklärung (BZgA) von Gesundheit Berlin-Brandenburg e. V. aufgebaut und betreut wird. Die einzelnen Projekte sollen besser miteinander vernetzt werden, um voneinander zu lernen und die Arbeit abzustimmen zu können. Hierfür wurden in den Bundesländern Koordinationsstellen, die sogenannten Regionalen Knoten eingerichtet. Über deren Arbeit wird hier laufend informiert.

Um die praktische Arbeit zu verbessern, werden im engen Austausch mit Vertreterinnen und Vertreter der Praxis nachprüfbare Kriterien für Projekte erarbeitet (vgl. Abbildung). Diese »Good-Practice«-Kriterien sollen als Maßstab für alle dienen. Praxisnahe Arbeitshilfen für Prävention und Gesundheitsförderung im Quartier unterstützen bei der Umsetzung.

Jährlich wird von Gesundheit Berlin-Brandenburg e. V. und zahlreichen Partnerorganisationen der Kongress Armut und Gesundheit organisiert. Informationen zum Kongress und zum aktuellen Stand der Vorbereitungen finden sich ebenfalls auf der Plattform. Allen Interessierten aus der gesundheitsfördernden Praxis, aus Politik, Wissenschaft und Gesundheitswesen stehen auf der Internet-Plattform darüber hinaus Gesetzestexte und Leitlinien zu politischen und rechtlichen Rahmenbedingungen und ein Überblick der aktuellen Forschungsarbeiten zur freien Verfügung. Ein Terminkalender informiert über themenbezogene Kongresse, Tagungen und Fortbildungen.

Internet http://www.gesundheitliche-chancengleichheit.de 